\title{
Distance-Decay Effect in Probabilistic Time Geography for Random Encounter
}

\author{
Zhang-Cai Yin ${ }^{1}{ }^{(\mathbb{O}}$, Zhang-Hao-Nan Jin ${ }^{1, *}$, Shen Ying ${ }^{2, *}{ }^{\mathbb{C}}$, Hui Liu ${ }^{1}$, San-Juan $\mathrm{Li}^{1}$ \\ and Jia-Qiang Xiao ${ }^{1}$ \\ 1 School of Resources and Environmental Engineering, Wuhan University of Technology, \\ Wuhan 430061, China; yinzhangcai@whut.edu.cn (Z.-C.Y.); lh469@whut.edu.cn (H.L.); \\ lisanjuan@whut.edu.cn (S.-J.L.); xiaojiaqiang@whut.edu.cn (J.-Q.X.) \\ 2 School of Resource and Environmental Sciences, Wuhan University, Wuhan 430061, China \\ * Correspondence: 219610@whut.edu.cn (Z.-H.-N.J.); shy@whu.edu.cn (S.Y.); \\ Tel.: +86-182-0273-2172 (Z.-H.-N.J.); +86-138-7140-5389 (S.Y.)
}

Received: 23 February 2019; Accepted: 1 April 2019; Published: 4 April 2019

\begin{abstract}
Probabilistic time geography uses a fixed distance threshold for the definition of the encounter events of moving objects. However, because of the distance-decay effect, different distances within the fixed threshold ensure that the encounter events do not always have the same possibility, and, therefore, the quantitative probabilistic time geography analysis needs to consider the actual distance-decay coefficient (DDC). Thus, this paper introduces the DDC and proposes a new encounter probability measure model that takes into account the distance-decay effect. Given two positions of a pair of moving objects, the traditional encounter probability model is that if the distance between the two positions does not exceed a given threshold, the encounter event may occur, and its probability is equal to the product of the probabilities of the two moving objects in their respective positions. Furthermore, the probability of the encounter at two given positions is multiplied by the DDC in the proposed model, in order to express the influence of the distance-decay effect on the encounter probability. Finally, the validity of the proposed model is verified by an experiment, which uses the tracking data of wild zebras to calculate the encounter probability, and compares it with the former method.
\end{abstract}

Keywords: time geography; distance-decay coefficient; space-time interaction; movement ecology; encounter probability

\section{Introduction}

Probabilistic time geography states that the encounter between two moving objects without observation is random. Given two observation points of a moving object or two adjacent control points in a space-time trajectory, time geography uses a space-time prism to represent the space-time uncertainty of the moving object between two given points. The intersection of a space-time prism and a plane at a certain moment, commonly referred to as a space-time disc, contains all of the reachable positions of the moving object at that moment. The position in the space-time disc is non-unique, and the moving object can only be in one position at a time, so the position of the moving object in the space-time disc is a random event. This randomness is expressed by probability density (PD) clouds in probabilistic time geography, which describes the probability of a moving object to each location in the disc. Furthermore, PDs can be used to measure the uncertainty of an encounter event when two moving objects are randomly located at their respective positions.

Winter and Yin [1,2] first developed the foundation for probabilistic time geography by modeling the PDs of moving objects through a random walk principle, and the encounter probability between 
moving objects with an overlap of PDs. Note that the positions of a pair of moving objects, A and B, are $a$ and $b$, respectively, and $p(a)$ and $p(b)$ are the probability of A and B visiting $a$ and $b$, respectively. In the discrete space, Winter and Yin [1] believed that if two moving objects are located in the same grid cell at the same time, it is possible for them to encounter each other, otherwise it is impossible. However, this method was based more on intuition and had some limitations, for example, A and B in the same cell cannot meet, because the distance between them is beyond their respective perceptual range. Recently, Downs [3] argued that the PD of a moving object can also be calculated by the inverse-distance-weighted method, and then proposed a method to calculate the probability of encounter based on a 3D voxel structure. In essence, Winter and Yin's approach is consistent with Downs's one. In terms of a PD model, although the random walk method is different from the inverse-distance-weighted method, the PD has a common feature, namely: the farther from the center, the smaller the probability is, which takes into account the distance-decay effect of PD. In terms of the encounter measure, although one is based on the areal unit in 2D and the other is based on the volumetric unit (voxel) in 3D, their encounter semantics are the same, that is, only two individuals located in the same unit can encounter. However, these encounter semantics neglect the individual's perception ability, and their application in continuous space will bring about the modifiable areal unit problem (MAUP), where the probability of encountering changes as the granularity of the discrete structure changes.

In order to improve the measurement of encounter uncertainty in continuous space, Yin et al. [4] proposed an encounter-probability measure model based on the distance relationship, which states that if the distance between two moving objects does not exceed the maximum range that the individual can perceive, their encounter is possible. This method of measuring the encounter probability uses the distance relationship between individuals, instead of the relationship of individuals in a discrete unit, so that it can be independent of the spatial data structure. This means that the method for continuous space can theoretically avoid MAUP when implemented with discrete structures. However, this method ignores the attenuation effects associated with distance, and thus the likelihood of encounter does not decrease with increasing the distance, with the result of overestimating the likelihood of encounter. According to Tobler's first law of geography [5], near things are more related to each other; this means that the intensity of interaction based on the distance measure should decrease with the increasing distance, which is independent of the type of moving object.

This paper develops a new method based on the distance-decay effect as a measure of the probability of encounter in continuous space. In theory, this method extends the distance-decay coefficient (DDC), based on Yins' former method. Given that two moving objects are located at two locations where the distance does not exceed a given threshold, the former method considers that the probability of encounter is equal to the product of the probabilities of two moving objects accessing their respective positions; in the proposed method, the resultant probability value is multiplied by the DDC in order to comprehensively measure the uncertainty of the encounter. Therefore, the proposed method is different from the traditional one. We will study the mathematical basis of the encounter probability measure method considering the distance-decay effect, and will develop the corresponding algorithm in a homogeneous space, which can also be extended to a constrained space [6].

The remainder of the paper is organized as follows: Section 2 provides an overview of the probabilistic time geography and random encounter in it, the distance-decay effect, and DDC. Section 3 constructs an encounter probability model based on the distance-decay effect. Sections 4 and 5 describe the proposed model's algorithm and its application results, respectively. Then, this paper closes with a discussion of the results and guidance for future work in Section 6. Finally, Section 7 summarizes the full paper. 


\section{Background}

\subsection{Probabilistic Time Geography and Random Encounter}

\subsubsection{Probabilistic Time Geography}

Probabilistic time geography is an extension of time geography based on probability. Given the space-time constraints of a moving object, including space-time trajectory control points (also known as anchor points), time geography considers the space-time position of the moving object between two anchor points to be uncertain [7]. The prism represents the uncertainty in the period between the two anchor points, while the space-time disc represents the reachable position range of the moving object at any time point. The events of the mobile object at each location in the space-time disc, hereafter referred to as location events, are random. Probabilistic time geography considers that the occurrence of location events is not always uniform, and provides a probability density cloud distributed over the disc as a sample space for location events.

In probabilistic time geography, some basic formulas, such as truncated normal distribution [2], truncated Brownian Bridge [8], and the Markov process [9], are used to estimate the position probabilities for vehicles [10], animals [3], and pedestrians [11], by using their trajectory data. As the probability density cloud can reflect the difference in the frequency of moving objects to different locations in the space-time disc, it can answer when and where a moving object is most likely to appear, and thus provides a basis for applications related to location probability. For example, it can be applied to person search and rescue [12], car accident analysis, and movement ecology research [3]. Probabilistic time geography not only focuses on the positional probability of a moving object, but also on the possibility of encountering between two moving objects.

\subsubsection{Random Encounter in Probabilistic Time Geography}

Random encounter in probabilistic time geography has been widely discussed in recent years. As a kind of dynamic interaction, the probability of moving objects' random encounters can be quantitatively calculated by the methods of probabilistic time geography, taking into account the heterogeneity of time and space. Downs et al. [3] first proposed a method for calculating the encounter probability in continuous space, whose implementation is based on discrete structures. This method is convinced that an encounter event can occur when two moving objects are only in one discrete unit (i.e., voxel), otherwise it is impossible. However, this approach results in a MAUP as a result of the dependence on discrete structures. In general, as the granularity of discrete voxels decreases, the resultant encounter-probability decreases. Thus, on the basis of this method, Yin et al. [4] proposed a new method that introduces the concept of an encounter-distance threshold. If the distance between two moving objects does not exceed the threshold, it is possible that they encounter, otherwise it is impossible. This definition of encounter semantics is different from that of Downs et al. [3], and is independent of the type of spatial data structure.

For two moving objects, named $\mathrm{H}$ and $\mathrm{G}$, their space-time discs are defined separately as $\Omega_{t}^{x}(x \in$ $\left.\Omega_{t}^{x}\right)$ and $\Omega_{t}^{y}\left(y \in \Omega_{t}^{y}\right)$ at a specific time $t$, where $x$ is a potential location for Hat time $t$ and $y$ is a potential location for $\mathrm{G}$ at time $t$. The encounter event can be calculated as follows:

$$
E_{\text {meet }}\left(\Omega_{t}^{x}, \Omega_{t}^{y}\right)=\left\{\left\langle x, y ; t>\|y-x\| \leq d_{\text {meet }}, x \in \Omega_{t}^{x}, y \in \Omega_{t}^{y}\right\}\right.
$$

where, $E_{\text {ment }}$ is a collection of encounter events of $H$ and $G$ at time $t, d_{\text {ment }}$ is the encounter distance threshold, and $\|-\|$ is the Euclidean distance operator between any two locations. It is worth mentioning that the determination of the distance threshold is related to the characteristics of the moving object and the geographical environment. The size of the threshold has great influence on the measurement of the encounter event. Taking into account the mutual exclusion between encounter events, the probability of encounter can be calculated as follows: 


$$
P_{\text {meet }}\left(\Omega_{t}^{x}, \Omega_{t}^{y}\right)=\sum_{x \in \Omega_{t}^{x}} \sum_{y \in \Omega_{t}^{y},\|y-x\| \leq d_{\text {meet }}} h(x ; t) \cdot g(y ; t)
$$

where $P_{\text {ment }}$ is the encounter probability of two moving objects at time $t, h(x ; t)$ represents the probability that object $\mathrm{H}$ is at point $x$ at time $t$, and $g(y ; t)$ represents the probability that object $\mathrm{G}$ is at point $y$ at the same time $t$.

It can be known from Formula (1) that the judgment basis of the encounter event, $\|y-x\| \leq d_{\text {meet }}$, is independent of the change of the distance. That is, within the threshold $d_{\text {meet }}$, the intensity of the likelihood of an encounter event does not decrease as the distance increases. Although Equation (2) can reflect the strength of the encounter, it ignores the effect of distance decay, with the result of overestimating the probability of encounter. Therefore, considering the distance-decay effect is helpful for improving the encounter probability measure.

\subsection{Distance-Decay Effect and Distance-Decay Function}

The distance-decay effect comes from the first law of geography [6] - "Everything is related, but the closer the distance, the closer the relationship is". When in a space-time interaction, it can be understood that the closer the distance between two moving objects, the stronger the interaction between them. The distance-decay effect can be divided into two levels [13], namely: macroscopic and microscopic. At the macroscopic level, because of the distance-decay effect, the interaction between two geographical entities is negatively correlated with the distance, under the premise that other factors are relatively stable. At the microscopic level, the distance-decay effect can be interpreted as the probability that a single object (e.g., animal or person) that moves to a different destination is inversely related to the distance, and the strength of the interaction between two moving objects is inversely related to the distance.

The distance-decay effect can be quantified into a variety of mathematical expressions that fit the distance-decay function [13-17]. Figure 1 shows several common types of distance-decay functions, including the power-law type and exponential type (Figure 1a), piecewise function (Figure 1b). A common feature of these functions is that the function value $f(d)$ changes in a non-incremental manner as the distance $d$ increases. As a different expression of the distance-decay effect, these functions have different characteristics or application specificities. For example, Östh et al. [18] used the exponential-type distance-decay function to study the commuting distance in Sweden, Nijkamp et al. [19] detailed the problem of selecting the power-law and exponential-type distance-decay function, Stepniak et al. [20] used linear regression to fit the distance-decay function to study the spatial agglomeration problem in spatial interactions, and Ladau et al. [21] analyzed the similarity between biological community in the form of piecewise distance-decay functions.

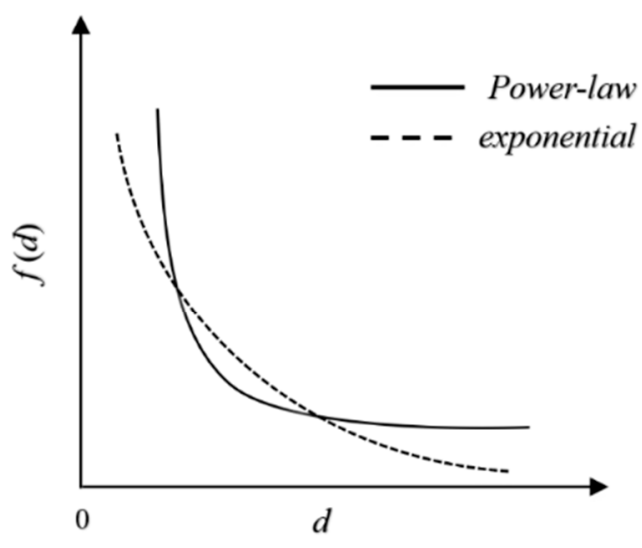

(a)

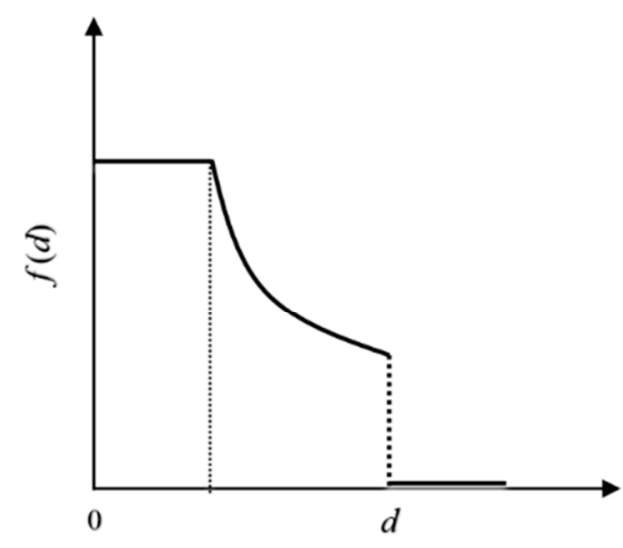

(b)

Figure 1. (a) Power-law and exponential distance-decay function; (b) piecewise distance-decay function. 
In recent years, distance attenuation effects have received constant attention in applications related to trajectory data, such as the similarity between plant community [21-23], spatial interaction between humans [24] or animals [25], and specific places. Some scholars have combined it with GIS in order to study the effects of environmental factors such as automobile exhaust on human diseases with distance attenuation [26,27]. However, most of these studies are focused on static interaction without temporal information, but can provide an application basis for the dynamic interaction with temporal information proposed in this paper.

\section{Random Encounter Model under Distance-Decay Effect}

The existing encounter probability measure model takes into account the temporal information, but ignores the distance-decay effect. Therefore, this paper combines the two in order to establish an encounter probability model based on the distance-decay effect.

\subsection{Construction of Encounter Event Based on the Distance Decay Effect}

From the perspective of GIScience, random encounter is a space-time phenomenon between two moving objects. This encounter is related to the distance between them, usually not exceeding the maximum perceived distance of both parties. Between the 0 and the encounter distance threshold (i.e., the maximum perceived range), potential encounter events of two moving objects are likely to occur. As a result of the existence of the distance attenuation effect, different distances will affect the uncertainty of the encounter. One measure of the magnitude of uncertainty is entropy. The greater the uncertainty, the greater the entropy, and vice versa. Thus, according to the first law of geography, the entropy of the encounter uncertainty changes in a non-decreasing manner as the distance changes from 0 to the distance threshold (Figure 2). As shown in Figure 2, rather than a constant weight " 1 " to all of the encounter distance within the distance threshold, the distance-decay function assigns a coefficient or weight of no more than 1 to different encounter distances, which could ensure that the closer encounter distance has a smaller entropy.

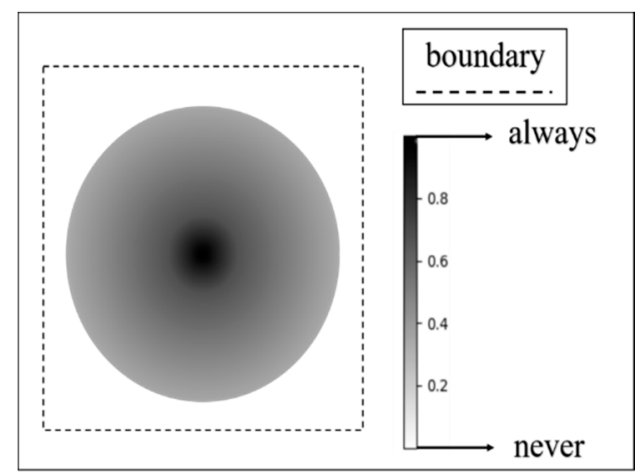

Figure 2. Illustration of the encounter event with distance-decay effect.

From the results of the uncertainty measure of the encounter event, the method that takes into account the attenuation effect will be different from the traditional method, with the result of a Boolean value of 0 or 1 . The result of the proposed method is a DDC between 0 and 1 , which distinguishes the difference in the actual distance between two objects. However, the traditional method only considers the upper bound of the distance, and does not factor in the actual distance. If the DDC is a constant 1 or the distance between two moving objects exceeds the encounter distance threshold, the proposed method is consistent with the conventional method; this means that the traditional method is a special case of the proposed method.

The encounter event based on the distance attenuation effect, $E_{\text {meet }}$, can be described as follows:

$E_{\text {meet }}\left(\Omega_{t}^{x}, \Omega_{t}^{y}\right)=\left\{<x, y ; t>\mid F(\|y-x\|)>0, x \in \Omega_{t}^{x}, y \in \Omega_{t}^{y}, t \in T\right\}$ 
$=\cup_{x \in \Omega_{t}^{x}} \underset{y \in \Omega_{t}^{y}}{\cup} E_{\text {meet }}(x, y ; t)=$

$$
\cup_{x \in \Omega_{t}^{x}} \underset{y \in \Omega_{t}^{y}}{\cup}\left([F(\|y-x\|)>0] \cdot E_{t}^{x} \cdot E_{t}^{y}\right)
$$

where $T$ is a tracking period, and $T=\left\{t_{1}, t_{2}, t_{3}, \ldots \ldots t_{n}\right\}$ with a time step; $F(d)$ is the distance-decay function with the result of a value between $[0,1] ; E_{\text {meet }}(x, y ; t)$ indicates that two moving objects $\mathrm{H}$ and $G$ at the point pair $(x, y)$ may meet at time $t$; and $E_{t}^{x}\left(\operatorname{resp} . E_{t}^{y}\right)$ is an event indicating that the object $H$ (resp. G) accesses the point $x$ (resp. $y$ ). Compared with Equation (1), the main difference of Equation (3) is to use DDC instead of the Boolean test, so Equation (3) not only considers the distance threshold $d_{\text {meet }}$ in Equation (1), but also quantifies the effect of the different distances within $d_{\text {meet }}$ on the interaction strength.

According to Equation (3), the Cartesian product of $\Omega_{t}^{x}$ and $\Omega_{t}^{y}, \Omega_{t}^{x} \times \Omega_{t}^{y}$ contains all of the position pairs of objects $H$ and $G$. The sample space of the encounter event is contained in $\Omega_{t}^{x} \times \Omega_{t}^{y}$, which is the set of position pairs that satisfy $F(\|y-x\|)>0, x \in \Omega_{t}^{x}, y \in \Omega_{t}^{y}$. Thus, if $E_{\text {meet }}(x, y ; t)$ occurs, then $(x, y) \in \Omega_{t}^{x} \times \Omega_{t}^{y}$, and vice versa. In probability theory, basic events are mutually exclusive; this means that the basic event $E_{\text {meet }}(x, y ; t)$ is mutually exclusive. Thus, $E_{\text {meet }}\left(\Omega_{t}^{x}, \Omega_{t}^{y}\right)$ is a collection of mutually exclusive events $E_{\text {meet }}(x, y ; t)$, that is, $E_{\text {meet }}\left(\Omega_{t}^{x}, \Omega_{t}^{y}\right)=\cup_{x \in \Omega_{t}^{x}} \cup \Omega_{t}^{y} E_{\text {meet }}(x, y ; t)$.

In addition, as the movement of $\mathrm{H}$ and $\mathrm{G}$ is considered to be independent, $E_{t}^{x}$ and $E_{t}^{y}$ are independent of each other. Thus, event $E_{\text {meet }}(x, y ; t)$ can be decomposed into the following form: $E_{\text {meet }}(x, y ; t)=[F(\|y-x\|)>0] \cdot E_{t}^{x} \cdot E_{t}^{y}$.

\subsection{Quantifying Distance-Decay Effect}

In this section, we need to solve the $F(d)$, which is discussed in a previous section and is defined in the encounter events. According to Long et al. [28], with research on encounter interactions, when the distance between moving objects exceeds a certain range (i.e., the maximum sensing range), the encounter between them is impossible; this means that there is an upper limit to the distance at which the encounter event occurs. Thus, above this upper bound, the attenuation coefficient is 0 ; otherwise, it is a positive number not exceeding 1 . In this way, it can be first concluded that the distance-decay function should be a piecewise function, and meanwhile, the domain of each segment function can be achieved as follows:

$$
F(d)= \begin{cases}1 & 0 \leq d \leq d_{l b} \\ f(d) & d_{l b}<d \leq d_{u b} \\ 0 & d>d_{u b}\end{cases}
$$

where $F(d)$ is called the distance-decay function in the total domain, $f(d)$ is a coefficient, $d$ is the distance between two moving objects, and $d_{l b}$ and $d_{u b}$ are two segmentation points in the total domain. When $d$ $>d_{u b}, F(d)=0$, this indicates that the encounter of two moving objects is not possible. When $d \in\left[d_{l b}\right.$, $\left.d_{u b}\right], F(d)=f(d)$; otherwise, $F(d)=1$. $d_{u b}$ corresponds to the $d_{\text {meet }}$ in Equation (1). Furthermore, if $f(d)=$ 1, Equation (4) is degenerated into a two-stage function; correspondingly, $F(\|y-x\|)>0$ in Equation (3) is degenerated to $\|y-x\| \leq d_{\text {meet }}$ in Equation (1). This also shows that Equation (1) is a special case of Equation (3).

Equation (4) provides a framework for the distance-decay function (e.g., Figure 3). In a specific application, the setting of the segmentation points and the selection of the segmentation function are related to the moving objects and the geographical environment.

Under the condition that the maximum sensing range of the moving object is known, the distance attenuation function can be obtained in two ways. One is the use of mathematical statistics, and the other is to use the common distance attenuation function mentioned earlier. Given the observation data of the potential interaction of two moving objects, the distance attenuation function is generated 
by the statistics of the interaction frequencies based on the different distances. This method has good application specificity, but requires a large amount of observation data, and it is difficult to apply to other environments, because the obtained attenuation function comes from a specific geographical environment. This paper uses a general distance attenuation function.

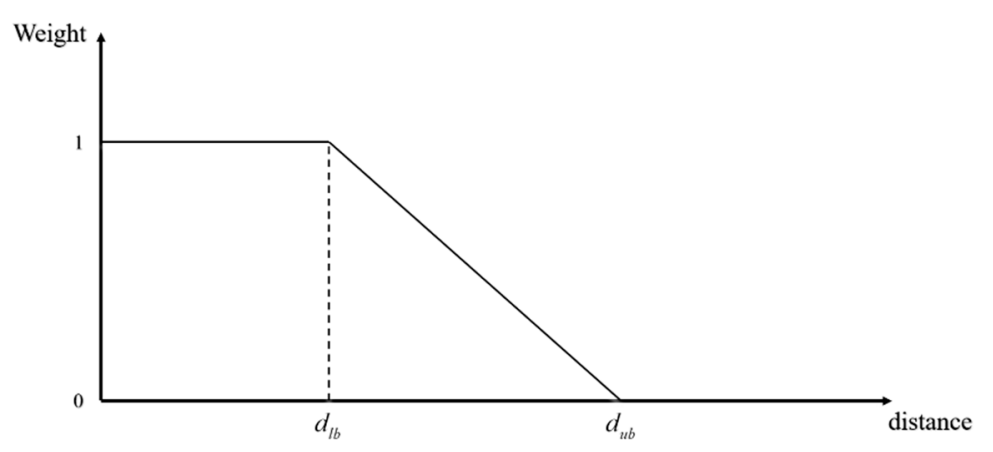

Figure 3. Illustration of a distance-decay function.

\subsection{Encounter Probability Model Based on the Distance-Decay Effect}

The probability of encounter is the probability of an encounter event between two moving objects. According to the function of $E_{\text {meet }}(x, y ; t)=[F(\|y-x\|)>0] \cdot E_{t}^{x} \cdot E_{t}^{y}$ in Equation (3), the probability of a basic event $E_{\text {meet }}(x, y ; t)$ can be calculated as follows:

$P_{\text {meet }}(x, y ; t)=P\left[E_{\text {meet }}(x, y ; t)\right]$ According to formula (3)

$=F(\|y-x\|) \cdot P\left(E_{t}^{x} \cdot E_{t}^{y}\right)$ Multiplication rule for independent events

$=F(\|y-x\|) \cdot P\left(E_{t}^{x}\right) \cdot P\left(E_{t}^{y}\right)=$

$$
F(\|y-x\|) \cdot h(x ; t) \cdot g(y ; t)
$$

where $F(\|y-x\|)$ is a coefficient not less than 0 , which is related to the distance $\|y-x\| ; h(x ; t)$ represents the probability that object $H$ is at point $x$ at time $t$; and $g(y ; t)$ represents the probability that object $\mathrm{G}$ is at point $y$ at the same time $t$. Thus, $P_{\text {meet }}(x, y ; t)$ represents the probability of encounter when moving objects $\mathrm{H}$ and $\mathrm{G}$ are at point pair $(x, y)$ at time $t$. As events $E_{t}^{x}$ and $E_{t}^{y}$ are independent of each other, the calculation of probability $P\left(E_{t}^{x} \cdot E_{t}^{y}\right)$ can be expressed as follows: $P\left(E_{t}^{x} \cdot E_{t}^{y}\right)=P\left(E_{t}^{x}\right) \cdot P\left(E_{t}^{y}\right)$, according to the multiplication rule of independent events.

Furthermore, according to the function of $E_{\text {meet }}\left(\Omega_{t}^{x}, \Omega_{t}^{y}\right)=\cup_{x y} E_{\text {meet }}(x, y ; t)$ in Equation (3), using Equation (5), the probability of $E_{\text {meet }}\left(\Omega_{t}^{x}, \Omega_{t}^{y}\right)$ can be calculated as follows:

$P_{\text {meet }}\left(\Omega_{t}^{x}, \Omega_{t}^{y}\right)=P\left(E_{\text {meet }}\left(\Omega_{t}^{x}, \Omega_{t}^{y}\right)\right)$ According to Formula (3)

$=P\left(\cup \cup E_{\text {meet }}(x, y ; t)\right)$ Addition rule for mutually exclusive events

$=\sum_{x \in \Omega_{t}^{x}} \sum_{y \in \Omega_{t}^{y}} P_{\text {meet }}(x, y ; t)$ According to Formula (5)

$$
=\sum_{x \in \Omega_{t}^{x}} \sum_{y \in \Omega_{t}^{y}} F(\|y-x\|) \cdot h(x ; t) \cdot g(y ; t)
$$

where $P_{\text {meet }}\left(\Omega_{t}^{x}, \Omega_{t}^{y}\right)$ represents the probability of encounter when moving objects $\mathrm{H}$ and $\mathrm{G}$ at time $t$. Equation (6), relative to Equation (2), introduces DDC, so that the uncertainty of the encounter interaction can be quantified by the DDC and position probabilities together. In addition, Equation (6) realizes the organic combination of the probability theory and perception-based encounter measures, thus providing a theoretical framework for the time-geographic measure of random encounters. For more complex scenarios, Equation (6) can also be extended, including introducing distance attenuation 
coefficients that vary over time and/or space, or constraints that are unlikely to meet at a particular time and/or space.

As DDC involves two variables, $d_{l b}$ and $d_{u b}\left(=d_{\text {meet }}\right)$, Equation (6) is not too sensitive to $d_{\text {meet }}$ with respect to Equation (2), only related to $d_{\text {meet }}$. In reality, the individual's maximum range of perception, $d_{\text {meet }}$, is often estimated based on a small number of samples, and is related to the specific geographical environment and the individuals, and thus has a strong uncertainty. In Equation (2), using $d_{\text {meet }}$ as the only indicator will obviously bring uncertainty to the measurement of the encounter event and to its probability. In DDC, the coefficients of the distance variable less than and close to $d_{\text {meet }}$ are set to a small value close to 0 , so that Equation (6), based on the DDC encounter-probability model, is not sensitive to the uncertainty of $d_{\text {meet }}$.

In addition, Equation (6) calculates the probability of encounter on a time slice $t$, so it can continuously calculate the probability of encounter at different times. For a tracking period of $T=\left\{t_{1}, t_{2}\right.$, $\left.t_{3}, \ldots \ldots t_{n}\right\}$, the probabilities of encounter at different times can be obtained, such as $P_{\text {meet }}\left(\Omega_{t_{1}}^{x}, \Omega_{t_{1}}^{y}\right)$, $P_{\text {meet }}\left(\Omega_{t_{2}}^{x}, \Omega_{t_{2}}^{y}\right)$, and so on, thus showing the trend of the probability of encounter in time. It is worth mentioning that the potential area (e.g., $\Omega_{t}^{x}$ ) of the moving object in Equation (6) can be replaced with a fixed spatial location point (e.g., $x$ ). An encounter probability for a given spatial location can be derived as follows:

$$
P_{\text {meet }}\left(x, \Omega_{t}^{y}\right)=\sum_{y \in \Omega_{t}^{y}} F(\|y-x\|) \cdot h(x ; t) \cdot g(y ; t)
$$

Furthermore, given that moving object $\mathrm{H}$ is located at $x$ point in period $T$, the probability that $\mathrm{H}$ can meet $\mathrm{G}$ can be calculated as follows:

$$
P_{\text {meet }}\left(x, \Omega_{t}^{y} ; T\right)=P_{\text {meet }}\left(x, \Omega_{t_{1}}^{y}\right) \cup P_{\text {meet }}\left(x, \Omega_{t_{2}}^{y}\right) \cup, \ldots \cup P_{\text {meet }}\left(x, \Omega_{t_{n}}^{y}\right)
$$

where $P_{\text {meet }}\left(x, \Omega_{t}^{y} ; T\right)$ is the probability that moving object $\mathrm{H}$ at point $x$ can meet the moving object $\mathrm{G}$ in period $T$. As $\mathrm{H}$ and $\mathrm{G}$ can have multiple encounters at different times in period $T$, the addition of Equation (8) can only use the probability addition formula of general events and cannot use that of mutually exclusive events. The mapping of variable $P_{\text {meet }}\left(x, \Omega_{t}^{y} ; T\right)$ to the $x$ point on the map can generate an encounter-probability map, which can show the spatial distribution characteristics of the encounter probability. Similarly, the probability of interaction between a pair of moving objects $\mathrm{H}$ and $G$ during the tracking period can be calculated as follows:

$$
P_{\text {meet }}\left(\Omega_{t}^{x}, \Omega_{t}^{y} ; T\right)=P_{\text {meet }}\left(\Omega_{t_{1}}^{x}, \Omega_{t_{1}}^{y}\right) \cup P_{\text {meet }}\left(\Omega_{t_{2}}^{x}, \Omega_{t_{2}}^{y}\right) \cup, \ldots \cup P_{\text {meet }}\left(\Omega_{t_{n^{\prime}}}^{x}, \Omega_{t_{n}}^{y}\right)
$$

\section{Application}

The encounter-probability algorithm based on the distance-decay effect is applied to the tracking data of zebras from an open-source data website (https://crawdad.org), which is on elaborated by Downs et al. [3] and Yin et al. [4]. This experiment selected data from three zebras, including a male zebra (labeled A) and two female zebras (labeled B and C), during the same period for $60 \mathrm{~h}$ from 12:00:00 on 24 June 2005 to 00:00:00 on 27 June 2005. This time period was chosen mainly because the tracking data of the three zebras during this period was relatively complete. These three zebras are from different populations and thus reflect the behavioral characteristics of their respective populations. Correspondingly, the interaction between the three zebras can also reflect the interaction characteristics between adjacent populations. It is worth mentioning that zebra $\mathrm{A}$ is a bachelor and shows a trend of courtship, which provides a biological basis for its interaction with the other two zebras. For the interaction of the three zebras, this experiment mainly analyzes the interaction between two zebras, and only considers the A-B interaction and A-C interaction. For illustration, the space-time trajectory data of the three zebras is visualized in the ArcScene 10.2 as shown in Figure 4. 


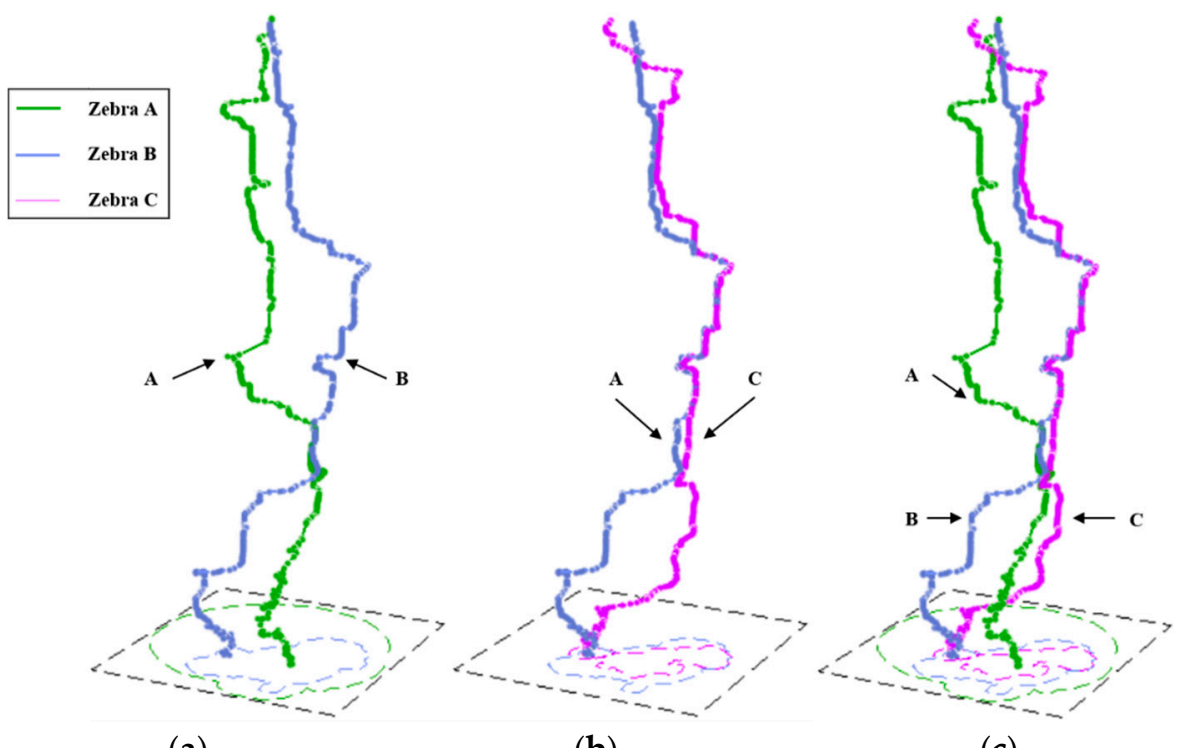

(a)

(b)

(c)

Figure 4. Trajectory data for three zebras: (a) A-B; (b) A-C; (c) A-B-C.

The potential area for each zebra is represented using the voxel-based model with time steps of $1 \mathrm{~min}$, a raster resolution of $120 \mathrm{~m}$, and a fixed maximum velocity of $1.5 \mathrm{~m} / \mathrm{s}$ [3,4]. According to the tracking data for each zebra, the average speeds between the adjacent track points are calculated by the distance between these two points divided by the corresponding elapsed time. The result shows that $99.7 \%$ of these average speeds do not exceed $1.5 \mathrm{~m} / \mathrm{s}$ for the three zebras, which means that $1.5 \mathrm{~m} / \mathrm{s}$ is large enough for this analysis. If too much speed is selected, the space-time disc proportional to the speed will contain many positions that are impossible for the moving object to reach, and thus the probability of non-zero will also be assigned to the unreachable position. Conversely, if too small a speed is selected, it will cause the time-space disc to repel the reachable position. Therefore, the choice of the maximum possible speed of a moving object depends not only on the type of moving object (such as species), but also on the geographical environment and individual characteristics. The space-time trajectory is the result of the interaction of a particular individual with a particular environment, specifically, the outcome of an individual with the maximum possible speed limited by a particular environment. In this sense, the maximum possible speed with trajectory derivation is application-specific, which systematically considers the dual characteristics of the individual and the environment. The inverse-distance-weight method is used to calculate the probability of each location in the corresponding potential area; thus, the magnitude of the probability that a moving object accesses a location is inversely related to the distance. In addition, the encounter distance threshold $d_{\text {meet }}$ was set to $200 \mathrm{~m}$, which was the maximum observed distance of zebras in the population [4].

Based on the above data and parameters, the calculation of the encounter-probability can be divided into two basic steps. First, determine the DDC. When the distance variable $d$ is in the range of $0-50 \mathrm{~m}$, the DDC is set to 1 ; in 50-200 m), the DDC is set to a straight-line segment between the two ends $(50,1)$ and $(100,0)$. For this illustration, this attenuation coefficient uses a simple linear segmentation model, where the upper bound $d_{\mathrm{ub}}=200 \mathrm{~m}$ is derived from the maximum sensing range. The breakpoint of $50 \mathrm{~m}$ is derived from the literature [28], which uses this value to measure the spatial proximity of the animals. Then, calculate the probability of encounter. According to Equation (6), the probability of interaction between a pair of zebras at any one time can be calculated; thus, the sequence encounter probability at a series of discrete moments can generate a graph of the probability of encounter with time. Furthermore, according to Equation (8), the probability that one zebra at a given position can encounter another zebra during a tracking period can also be generated, and a map of the probability of encounter can be created by mapping these resultant probabilities to their corresponding positions. 


\section{Results}

Figure 5 illustrates maps of the probability of encounter that take into account the effects of distance attenuation. Of them, Figure 5a,c shows the probability maps of the interaction of zebra A with B and C, respectively, during the tracking period. In order to show the impact of the DDC introduction on the encounter probability, Figure $5 b$,d show the encounter-probability maps without DDC, respectively. From these maps, there is little difference between the maps with DDC and those with no DDC, especially for the spatial extent of the probability distribution. The biggest difference is the probability density on the map. From the comparison of the A-B and A-C interaction probability maps, it can be seen that the intensity and breadth of the A-B interactions are significantly larger than the A-C interactions, so we can also conclude that A-B has dynamic interactions.

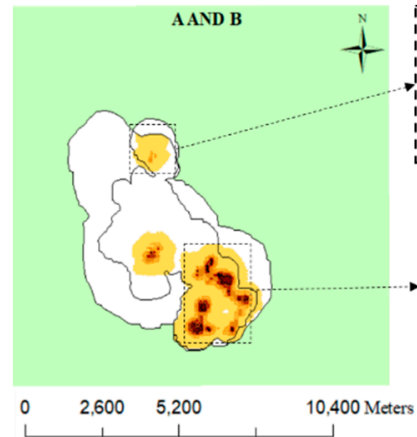

(a)

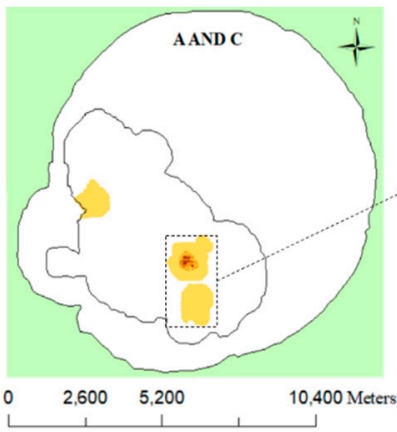

(c)

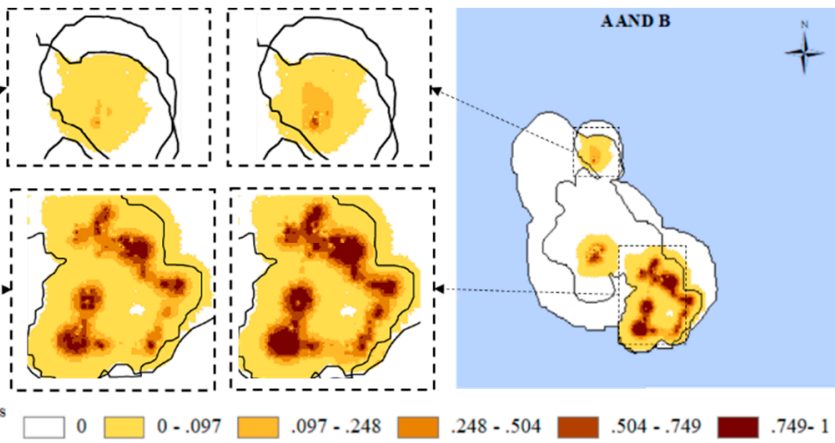

(b)

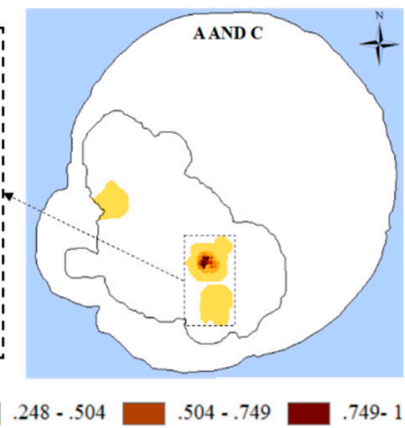

(d)

Figure 5. Encounter probability maps with the distance-decay coefficient (DDC) (a,c) and without the $\operatorname{DDC}(\mathbf{b}, \mathbf{d})$.

While the probability map shows the encounter probability over the spatial distribution, a probability histogram is also generated to detail the probability over time. As shown in Figure 6a,b, the strength and duration of the interaction of $A$ to $B$ is greater than the interaction of $A$ and $C$. Day one-zebra A interacted with B and did not interact with C. Day two-zebra A has a strong interaction with $C$ but has a short duration, while interaction with B lasts for a long time with strong intensity. Day three-zebra A has no interaction with $C$, while its interaction with $B$ changes from strong to weak. In general, the male zebra A was seeking interactive female zebras, and after a brief contact with zebra $C$, finally chose a substantial interaction with zebra B. According to the variation diagram of encounter probability with time, zebras A and B were attracted to each other, while zebras A and $\mathrm{C}$ tended to repel each other. Despite the different expressions, maps and histograms are reflections of the probability of encounter, and both show that the intensity of the A-B interaction is higher than that of A-C. In addition, zebra A interacts with at most one zebra at a time during the tracking period, which provides a basis for the analysis of dynamic interactions between multiple organisms in movement ecology. 

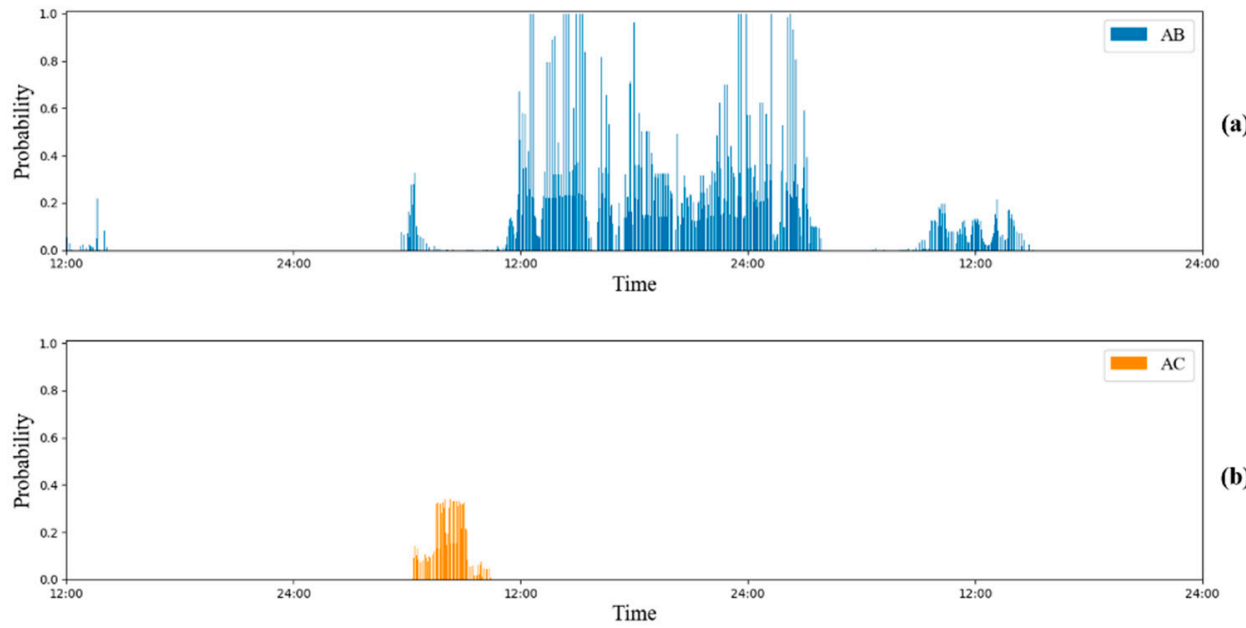

(b)
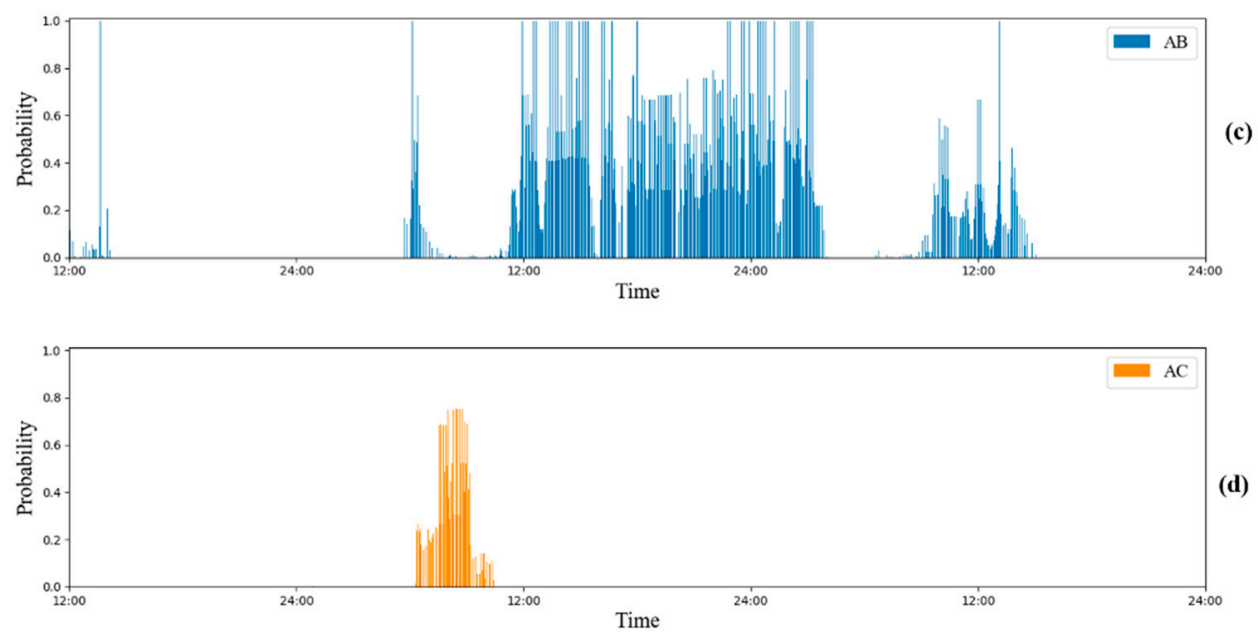

Figure 6. The probability histograms of $A B$ and $A C$ zebras over a 60-h period: (a) A-B and (b) A-C from the model with DDC; (c) A-B and (d) A-C from the model without DDC.

In order to further compare the differences between the two encounter-probability models with DDC and no DDC, based on the same parameters and data, the traditional encounter model is used to generate the change of the encounter probability with time (Figure $6 c, d$ ). As a result of the existence of the distance attenuation effect, the histogram generated by the proposed model avoids an excessive pulse-type encounter probability with respect to the traditional model, and is smooth and hierarchical overall. This difference can be attributed to the difference between the DDC and the constant 1 used in the traditional model, which considers that the interaction strength does not decrease with increasing distance (i.e., $\mathrm{DDC}=1$ ).

The above example uses the zebra data to test the effectiveness of the proposed model instead of the traditional model. Although the model proposed in this paper is only validated based on zebra data, it is also applicable to the space-time trajectory data of other objects, including other species of wildlife and other types of moving objects.

\section{Discussion}

This paper constructs an encounter probability model based on the distance attenuation effect, which takes into account the first law of geography compared to the traditional model. The implementation of the proposed model involves many parameter settings, such as the unit size of the discrete structure, the threshold of the maximum encounter distance, the distance attenuation coefficient. Most of them are discussed in the existing literature (e.g., [3,4]). The setting of the DDC function is mainly discussed here. The DDC replaces constant 1 , so the proposed model is more flexible 
and adaptable; however, the DDC increases a coefficient function and its variable $d_{\mathrm{lb}}$ compared to the single $d_{\text {meet }}$ in the traditional model, so the settings of the variables and their functions need to be considered when applying the proposed model.

First, determine the appropriate values for the $d_{\mathrm{lb}}$ and $d_{\mathrm{ub}}$ parameters in the DDC function, which affect the measure of the probability of encounter. The DDC is a complex function, which is related to moving objects, geographical environment, and research objectives. The maximum perceived distance $d_{\mathrm{ub}}$ of different types of moving objects is different. In movement ecology, the $d_{\mathrm{ub}}$ usually comes from the maximum distance between the different wild animals in the same population. Through experimental observation, the maximum spacing of lions in the lion group and the maximum spacing of the wild deer in the deer group are $200 \mathrm{~m}$ and $50 \mathrm{~m}$, respectively [4]. This distance is suitable for the identification of whether different individuals belong to the same population, but is not suitable for interaction between different species (such as predation behavior), and thus cannot replace the $d_{\mathrm{ub}}$ of the individuals in the interaction of avoiding natural enemies. In the DDC function, the maximum interval between individuals within the same population can be used as $d_{\mathrm{lb}}$, and the maximum perceived distance of the individual can be used as $d_{\mathrm{ub}}$. In addition, according to the specific geographical environment and research objectives, the two parameters $d_{\mathrm{lb}}$ and $d_{\mathrm{ub}}$ can be adjusted appropriately.

Next, determine the type of DDC function. There are many types of distance attenuation functions, each with their advantages and application specificity. However, the DDC function can usually be divided into two types-linear and nonlinear (such as power function or exponential function). Nevertheless, different types of DDC functions are not significantly different under the same conditions of $d_{\mathrm{lb}}$ and $d_{\mathrm{ub}}$-point $(0,1)$ and point $\left(d_{\mathrm{lb}}, 1\right)$ are usually connected as a horizontal straight line segment, and a certain curve (the straight line is the simplest curve) is used to interpolate between point $\left(d_{\mathrm{lb}}, 1\right)$ and point $\left(d_{\mathrm{ub}}, 0\right)$. The accurate acquisition of the DDC functions requires a large amount of spatiotemporal trajectory data and observation data for dynamic interactions in order to fit and correct the DDC functions through the frequency statistics of interactions. An approximate DDC function can use the universal distance attenuation function for reference and can be modified appropriately in combination with specific applications.

Then, regress and verify the DDC. The distance attenuation effect is a law and is therefore theoretically independent of the specific application. When the value of the $d_{\mathrm{ub}}$ variable is increased, the encounter event and probability that the proposed model can contain will also change in an undiminished manner. Moreover, this increase is convergent. When the $d_{\mathrm{ub}}$ is set to a sufficiently large value, the distance between the two moving objects will not exceed this value as a threshold, and is therefore considered to be always possible to meet, and the probability of encounter is always 1 . For this illustration, the value of $d_{\mathrm{lb}}$ is fixed so as to simply analyze the distance attenuation phenomenon of the encounter probability as a function of $d_{\mathrm{ub}}$. For $d_{\mathrm{ub}}$ to uniformly sample nine points in the interval of (200-1000 m), Equation (9) is used to calculate the cumulative encounter-probability of zebra A and B during the tracking period. Figure 7a shows the average of the cumulative probability as a function of $d_{\mathrm{ub}}$; Figure $7 \mathrm{~b}$ shows the derivative of the function, which expresses the characteristic that the increase (or variation) of the encounter-probability decreases as the distance $d_{\mathrm{ub}}$ increases, and thus can be used for the correction and verification of the DDC function.

Finally, apply the encounter probability to analyze the dynamic interaction mode. In this paper, the distance-decay effect is applied to a space-time interaction such as a dynamic interaction. However, how to determine the type of dynamic interaction is one of the hot research issues in movement ecology nowadays [29]. In the case analysis, the encounter probability maps and histograms generated by the encounter probability model can be used to analyze the patterns of dynamic interaction, including mutual independence, mutual exclusion, or attraction. The encounter probability map and the histogram bridge the encounter probability model and the dynamic interaction model. Future work will develop an algorithm to determine the interaction mode using the encounter probability based on the space-time trajectory data. In addition, the encounter probability map is not a probability density function, because the sum of the probability on the map is generally not 1 . The events in 
which zebra A encounters zebra B at any position are non-mutually exclusive. Such events can occur in multiple places during the tracking period, and their respective probabilities can be equal to 1 , so that the sum of the encounter probabilities on the map is greater than 1. Although the sum of the probabilities on the map can be equal to 1 by the normalization method, the normalized map will be difficult for comparing the difference in the interaction strength between the A-B group and the A-C group. Another work in the future is to normalize the probabilities on the two maps as a whole, which not only facilitates the comparison between the two groups of interactions, but also uniformly expresses the interaction probabilities of zebra $\mathrm{A}$ in the different groups.
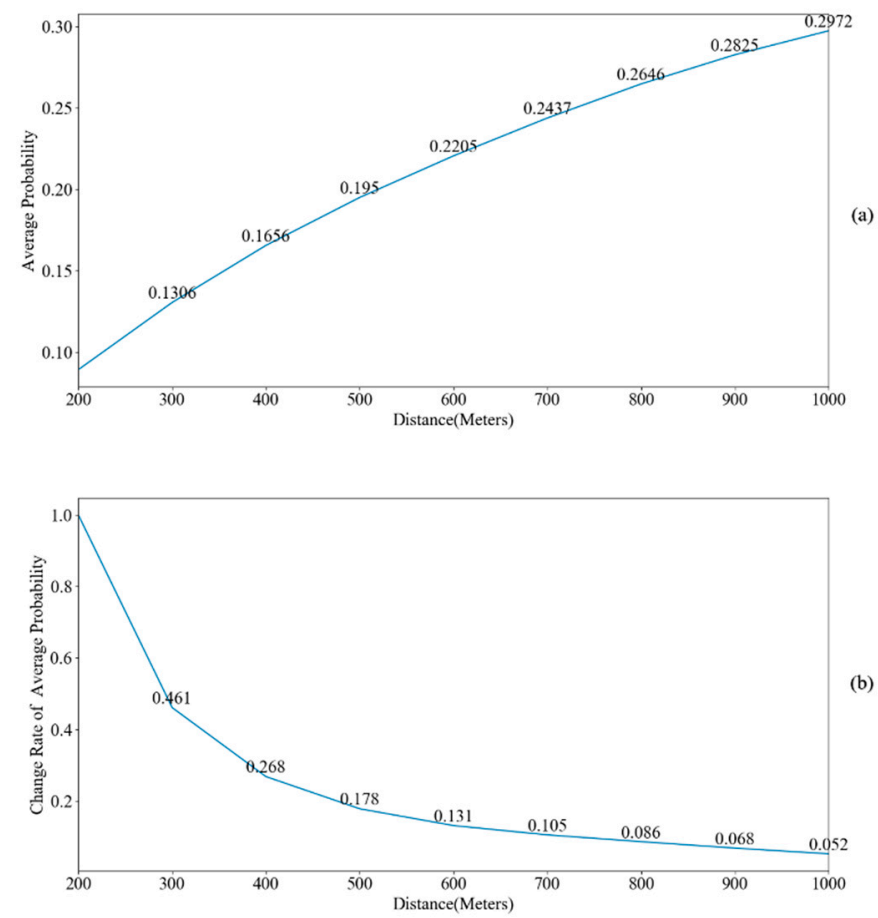

Figure 7. Attenuation of the probability of encounter with distance: (a) the average cumulative probability increases with distance; (b) the cumulative probability increment decreases with distance.

\section{Conclusions}

In this paper, an encounter-probability model based on the distance-decay effect has been introduced, which uses the distance attenuation coefficient instead of the constant 1 in the traditional encounter model. As constant 1 is a special case of distance attenuation coefficient, the proposed model is also a generalization and unification of the traditional model. In the case study, the proposed model can generate the encounter probability and its change graph with time and space, based on the space-time trajectory data of moving objects, and it can also support the analysis of dynamic interaction patterns between moving objects. In addition, in order to compare the effect of the improvement, the proposed model is compared with the traditional model, including the encounter probability map and the encounter probability histogram. The results show that the proposed model exhibits smooth and continuous characteristics on the encounter probability map and histogram compared with the traditional model, which is consistent with the continuous characteristics of the interaction behavior of wild zebras on the grassland in time and space. Finally, the distance-decay effect of the encounter behavior is simulated by the model proposed in this paper, which provides a basis for the regression analysis of the distance attenuation coefficient.

However, several limitations and improvements should be discussed in future research. First of all, although the implementation of the proposed model is independent of the spatial data structure type, its application in the real environment is based on a discrete structure. As with any discrete model, the smaller the size of the discrete unit, the higher the accuracy of the model shows, but this 
also increases the computational complexity. In the future, we will study how to choose an appropriate spatial granularity based on the specific application. Next, in the case analysis, this paper uses the inverse-distance-weight method to calculate the position probability, but this method ignores spatial heterogeneity. Future research will provide a position probability method that takes into account spatial differentiation. Then, applying the framework provided by the common law of distance attenuation function in different fields, combined with the encounter problem, how to construct the application-specific encounter-distance attenuation function is a problem that needs to be solved in future research. Finally, as an alternative to the traditional encounter model, how the proposed model is applied to measure the dynamic interaction mode of moving objects is another direction of future research.

Author Contributions: Conceptualization, Zhang-Cai Yin and Zhang-Hao-Nan Jin; methodology, Zhang-Cai Yin and Zhang-Hao-Nan Jin; software, Zhang-Hao-Nan Jin; validation, Zhang-Hao-Nan Jin; formal analysis, Zhang-Cai Yin and Zhang-Hao-Nan Jin, and Hui Liu; investigation, San-Juan Li; resources, Jia-Qiang Xiao; data curation, Zhang-Hao-Nan Jin; writing (original draft preparation), Zhang-Hao-Nan Jin; writing (review and editing), Zhang-Cai Yin, Zhang-Hao-Nan Jin, and Shen Ying; supervision, Zhang-Cai Yin; project administration, Zhang-Cai Yin; funding acquisition, Zhang-Cai Yin and Shen Ying.

Funding: This study was funded through support from the National Key R\&D Program of China (grant numbers 2017YFB0503700 and 2017YFB0503500), and the National Science Foundation of China (NSFC) (grant numbers 41671381 and 41531177).

Acknowledgments: The completion of this article is also inseparable from the support of my classmates.

Conflicts of Interest: The author declares no conflict of interest.

\section{References}

1. Winter, S.; Yin, Z.C. The elements of probabilistic time geography. GeoInformatica 2011, 15, 417-434. [CrossRef]

2. Winter, S.; Yin, Z.C. Directed movements in probabilistic time geography. Int. J. Geogr. Inf. Sci. 2010, 24, 1349-1365. [CrossRef]

3. Downs, J.A.; Horner, M.W. Voxel-based probabilistic space-time prisms for analysing animal movements and habitat use. Int. J. Geogr. Inf. Sci. 2014, 28, 875-890. [CrossRef]

4. Yin, Z.C.; Wu, Y.; Winter, S. Random encounters in probabilistic time geography. Int. J. Geogr. Inf. Sci. 2018, 1-17. [CrossRef]

5. Tobler, W.R. A computer movie simulating urban growth in the Detroit Region. Econ. Geogr. 1970, 46, 234-240. [CrossRef]

6. Yu, W.H.; Chen, Y.G. Regional co-location pattern scoping on a street network considering distance decay effects of spatial interaction. PLoS ONE 2017, 12, e0181959. [CrossRef] [PubMed]

7. Miller, H.J.; Bridwell, S.A. A field-based theory for time geography. Ann. Assoc. Am. Geogr. 2009, 99, 49-75. [CrossRef]

8. Song, Y.; Miller, H.J. Simulating visit probability distributions within planar space-time prisms. Int. J. Geogr. Inf. Sci. 2014, 28, 104-125. [CrossRef]

9. Song, Y.; Miller, H.J. Modeling visit probabilities within network-time prisms using markov techniques. Geogr. Anal. 2016, 48, 18-42. [CrossRef]

10. Downs, J.A.; Horner, M.W. Probabilistic potential path trees for visualizing and analyzing vehicle tracking data. J. Transp. Geogr. 2012, 23. [CrossRef]

11. Abi-Zeid, I.; Frost, J.R. SARPlan: A decision support system for canadian search and rescue operations. Eur. J. Oper. Res. 2005, 162, 630-653. [CrossRef]

12. Demvsar, U.; Buchin, K. Analysis and visualisation of movement: An interdisciplinary review. Mov. Ecol. 2015, 3, 1-24. [CrossRef]

13. Liu, Y.; Gong, L. Quantifying the distance effect in spatial interactions. Acta Sci. Nat. Univ. Pekin. 2014, 50, 526-534. [CrossRef]

14. Hipp, J.R.; Boessen, A. The shape of mobility: Measuring the distance decay function of household mobility. Prof. Geogr. 2016, 69, 1-13. [CrossRef]

15. McLaren, Z.M.; Ardington, C. Distance decay and persistent health care disparities in South Africa. BMC Health Serv. Res. 2014, 14, 541. [CrossRef] 
16. Fotheringham, A.S. Spatial structure and distance-decay parameters. Ann. Assoc. Am. Geogr. 2010, 71, 425-436. [CrossRef]

17. Halás, M.; Klapka, P. Distance-decay functions for daily travel-to-work flows. J. Transp. Geogr. 2014, 35, 107-119. [CrossRef]

18. Östh, J.; Lyhagen, J. A new way of determining distance decay parameters in spatial interaction models with application to job accessibility analysis in Sweden. Eur. J. Transp. Infrastruct. Res. 2016, 16, 344-363. [CrossRef]

19. Nijkamp, P.; Rietveld, P. Exponential or power distance-decay for commuting? An alternative specification. Environ. Plan. A 2004, 41, 461-480. [CrossRef]

20. Stępniak, M.; Jacobs-Crisioni, C. Reducing the uncertainty induced by spatial aggregation in accessibility and spatial interaction applications. J. Transp. Geogr. 2017, 61, 17-29. [CrossRef]

21. Ladau, J.; Green, J.L. The geometry of the distance-decay of similarity in ecological communities. bioRxiv 2017. [CrossRef]

22. Soininen, J.; Mcdonald, R. The distance decay of similarity in ecological communities. Ecography 2007, 30, 3-12. [CrossRef]

23. Hélène, M.; Chuyong, G. A general framework for the distance-decay of similarity in ecological communities. Ecol. Lett. 2008, 11, 904-917. [CrossRef]

24. Liu, Y.; Sui, Z.W. Uncovering patterns of inter-urban trip and spatial interaction from social media check-in data. PLoS ONE 2014, 9, e86026. [CrossRef]

25. Luncz, L.V.; Proffitt, T. Distance-decay effect in stone tool transport by wild chimpanzees. Proc. R. Soc. B Biol. Sci. 2016, 283. [CrossRef]

26. Requia, W.J.; Roig, H.L. Mapping distance-decay of cardiorespiratory disease risk related to neighborhood environments. Environ. Res. 2016, 151, 203-215. [CrossRef]

27. Requia, W.J.; Petros, K. Mapping distance-decay of premature mortality attributable to $\mathrm{PM}_{2.5}$-related traffic congestion. Environ. Pollut. 2018, 243, 9-16. [CrossRef]

28. Long, J.A.; Webb, S.L. Mapping areas of spatial-temporal overlap from wildlife tracking data. Mov. Ecol. 2015, 3, 38. [CrossRef]

29. Zhang, P.D.; Beernaerts, J. A hybrid approach combining the multi-temporal scale spatio-temporal network with the continuous triangular model for exploring dynamic interactions in movement data: A case study of football. Isprs Int. J. Geo-Inf. 2018, 7, 31. [CrossRef] 